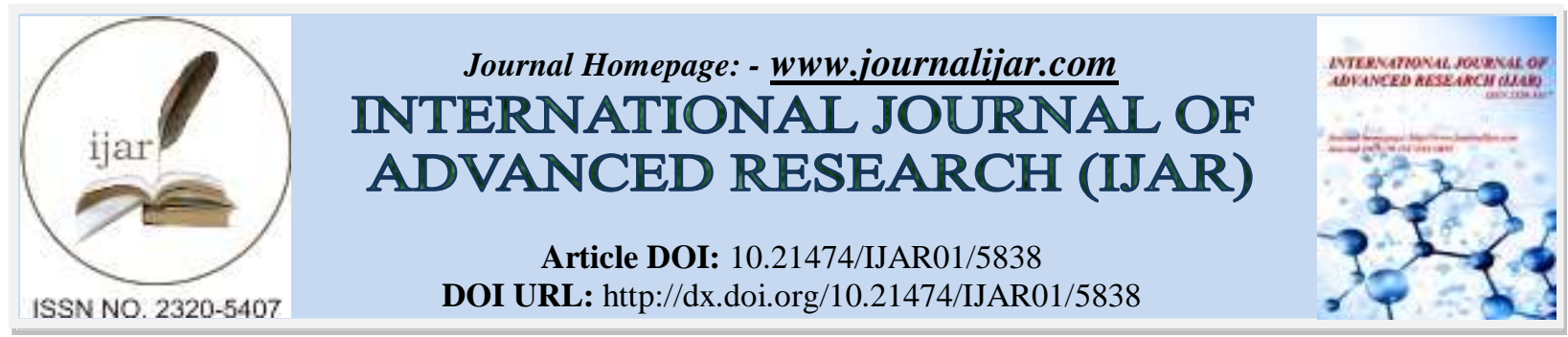

RESEARCH ARTICLE

\title{
ON ANALYSIS AND EVALUATION OF COCKTAIL PARTY EFFECT ON APPLIED EDUCATIONAL PRACTICE THEORY USING NEURAL NETWORKS MODELING.
}

Hassan M. H. Mustafa and Fadhel Ben Tourkia

Comp. Eng. Dept., Al-Baha Private College of Sciences, KSA.

\section{Manuscript Info}

Manuscript History

Received: 11 September 2017

Final Accepted: 13 October 2017

Published: November 2017

Key words:-

Artificial neural network modeling, noisy crowded learning environment, signal to noise ratio, cocktail party problem.

\section{Abstract}

This piece of research deals with an interestingly observed interdisciplinary challenging educational issue associated with children's learning performance phenomenon in classrooms. more precisely, it addresses an answer for the critically challenging educational question : how any of the students could focus on teachers' interactive speaking in noisy environmentally overcrowd class?. more precisely. More specifically, it deals with the critical educational issue of What is the effect of the school environment on students considering Cocktail Party Effect (CPE) on learning achievement and its relation to the Educational Practice Theory. Moreover, this work presents a systematic approach for evaluation of an interdisciplinary phenomenal problem of human's selectivity auditory scene analysis. Interestingly, in the educational field practice this Cocktail Party Effect results in the Cocktail Party Problem (CPP) dealing with an auditory perception phenomenon. Additionally, it is noticed that introduced proposal for active audition modeling is motivated by analogous active vision processes, such as that observed during Optical Character Recognition (OCR). In nature, observed OCR as well as pattern recognition processes have to be carried out under non ideal environmental learning condition (under effect of noisy data). More particularly, CPP is motivated to be solved by adopting selective auditory attention or equivalently selective hearing which is a type of selective attention and involves the auditory system of the nervous system. Accordingly, children's Selective hearing is characterized as the action in which considerable children's ability to focus their attention inside classroom on a specific source of teacher's spoken wording signals. Commonly, this process experienced as following one speaker (teachers' speech) in the presence of another noisy overcrowded signals resulting in CPE at classroom. By the end of this paper, some interesting simulation results presented after taking into account the comparative studies of two essential ANN parameters namely : learning rate and gain factor values. Versus varying neurons' number of the hidden layer associated to self-organized ANN paradigm model. These results revealed the effect of interrelation between various learning rate values against different values of signal to noisy ratio considering student's selective responsive attention.

Copy Right, IJAR, 2017,. All rights reserved. 


\section{Introduction:-}

The field of the learning sciences is represented by a growing community internationally. The last decade of previous century(1990-2000) named as the Decade of the brain, after referring to WHITE HOUSE OSTP REPORT (U.S.A.) which declared in 1989 [1]. Therefore, educationalists as well as computer engineering scientists have adopted research approach associated with natural intelligence (recent computer generation),and basic brain functions (learning and memory). Furthermore, many educational experts have currently recognized the conventional ways of conceiving learning are facing increasing challenges in this time of rapid Technological, and social changes [2][3][4]. Consequently, building up realistic simulation for modeling various human brain functions have been adopted by educational experts as a relevant interdisciplinary evolutionary trend. Therefore, building up realistic simulation for modeling various human brain functions have been adopted by educational experts as a relevant interdisciplinary evolutionary trend. That is by incorporating learning sciences with Nero-physiology, Psychology, and Cognitive science in order to investigate systematically some increasing challenging interdisciplinary issues. Specifically, this paper considers the increasingly sophisticated role of Artificial Neural Networks $\left(\mathrm{ANN}^{\mathrm{S}}\right)$ concerned with environmental learning phenomenal issues associated to an interdisciplinary discipline incorporating neuroscience, education, and cognitive sciences. Consequently, Neural Networks theorists as well as educationalists and neurobiologists have focused their main attention on reaching innovative investigational contributions. That is for solving the critical problematic question: how student's brain can perform well learning function considering noisy environmental conditions? In the context of Artificial Neural Networks $\left(\mathrm{ANN}^{\mathrm{s}}\right)$, that educational field critical question has to be mapped into two interrelated questions: how realistic simulation using ANN modeling capable to evaluate learning process convergence considering noisy environment? $\&$ how this process may be quantitatively affected by noisy contaminated data provided to students via noisy teacher's speaking signals? Interestingly, students' educational performance problem agrees (analogously) with the observed phenomenon in the context of communication field engineering. Therein, the ratio of the power or volume (amplitude) of a desired signal to the amount of mixed disturbances the noise observed due to cocktail party, contaminating that desired signal's power. This ratio is defined as signal-to-noise ratio abbreviated as SNR or S/N which measures the clarity of the received desired signal through any transmission/communication channel. Furthermore, in analog and digital communications, ratio, often SNR is a measure of signal strength relative to background noise [2]. Herein, a self-organized unsupervised ANN model has been suggested, for measuring selective performance which focused on attention and recognition for auditory signals. That focusing process is in well analogy to visual signal focusing to optical character recognition (OCR) that contaminated by intended various noisy power levels (signal to noise ratios). Furthermore, the suggested ANN simulation model obeys the brain targeted teaching model [5][6] and it agrees with the competitive learning law introduced by either Kohonen or Hebbian rule as well [4][5].

In more details, this research work has an interdisciplinary characteristic that specifically addresses a study of an educational challenging issue facing students in classrooms. In the context of solving of Pattern recognition problem concerned with educational field. The presented study gives a special attention of how machines (such as computers) can observe the environment, learn to distinguish patterns of interest (Characters) from their natural noisy background [4]. These observations resulted in a sound evaluation, and reasonable proper decisions considering patterns' data recognition (Optical Character Recognition- O.C.R.) modulated by noisy environmental contamination. Furthermore, this study associated with evaluation and analysis of learning performance considering noisy environmental conditions [7][8]. Generally, in classrooms noisy data considered as main cause of environmental annoyance and it negatively affects the quality of life of a large proportion of the population (students in classroom). More specifically, in our schools, these noisy conditions contaminate information/data which vulnerable to two types of contaminating noise: either external or internal (inherent). This paper pays a special attention to the internal (inherently) contaminated information/data that resulted by acquired noise via lessons. Accordingly, this inherently environmental CPE noise resulted in deteriorated learning achievements academic achievements (outcomes) of students facing noisy environmental CPE in classrooms.

The rest of this paper is organized as follows. at the two next second and third sections, revising of practice theory and modeling of educational interactive process are respectively, introduced. A brief revising of cocktail party problem (C P P ), in addition to a brief description of classroom noise effect on students' academic achievements are presented respectively at the fourth and fifth sections fifth section. At the sixth section, some of obtained 
interesting simulation results are introduced. finally, some interesting conclusive remarks are given at the seventh section.

\section{Practice Theory:-}

Educational Noisy Environment

This article examines a critical issue related to the classroom noisy learning environment in educational field practice. The noise in classrooms observed to have a negative reactive effect on students academic achievements (equivalently learning quality). In such classrooms, findings associated to noisy environment shown to be originated in accordance with mutual interaction among students as the largest effective noise source on learning quality [9][10]. That interactive mutual noisy phenomenon is at well correspondence to the Cocktail Party phenomenon's negative effect on students academic achievements. In other words, students' negative mutual reaction to the effective noise observed during there listening effort, concentration difficulty and interference irritation in learning process which results in deteriorated learning outcomes(grades). Interestingly, this research work motivated by ANN modeling paradigms relevant to educational applications in practice (at classrooms). Additionally, motivated by the fact of overwhelming majority of neuroscientists have adopted the concept suggests that huge number of neurons constituting the central nervous system (CNS). Considering, the synaptic interconnectivity performing dominant roles for learning processes in mammals besides humans [11]. More specifically, this motivation is supported by what revealed by National Institutes of Health (NIH) in U.S. that children in elementary school, may be qualified to learn "basic building blocks" of cognition and that after about 11 years of age, children take these building blocks and use them [12][13]. The extremely composite biological structure of human brain results in everyday behavioral learning brain functions. At the educational field, it is observed that learning process performed by the human brain is affected by the simple neuronal performance mechanism [14]. In this context, neurological researchers have recently revealed their findings about increasingly common and sophisticated role of Artificial Neural Networks $\left(\mathrm{ANN}^{\mathrm{s}}\right)$. Mainly, this role has been applied for systematic and realistic modeling of essential brain functions (learning and memory)[15].Accordingly, neural network theorists as well as neurobiologists and educationalists have focused their attention on making interdisciplinary contributions to investigate the observed educational phenomena associated with brain functional performance such as optimality of learning processes [16][17].Therefore, an unsupervised ANN model has been suggested herein, to measure performance of selective attention and recognition for visual signal specifically optical character recognition (OCR) subjected to various contaminating noisy levels (Signal to noise ratios). Finally, obtained simulation results declared the effect of Neural Network's parameters' relation between extrinsic \{various noisy levels (corresponding learning rate values) \} and intrinsic $\{$ individual students' differences (gain values) \} factors on recognition and selective attention performances. Additionally, presented obtained findings proved to be in well agreement with recently published results considering the dealing with noisy environmental learning problem [8][9]. Since beginning of last decade, Artificial Neural Networks $\left(\mathrm{ANN}^{\mathrm{S}}\right)$ models have been adopted to investigate systematically mysteries of human brain, the most complex biological neural system, [1][18]. In this context, there is strong evidence in modern neuroscience that networks of neurons perform a dominant role in performing cognitive brain functioning such as selectivity. Consequently, ensembles of highly specialized neurons (neural networks) in human play the dominant dynamical role in the functioning of developing selectivity function by brain [19][20][21][22].

\section{Recent Educational Practice Theory:-}

Researchers and designers of learning environments often debate whether the learner should adapt to the learning environment or whether the learning environment should adapt to them. Arguably this is the wrong question. A better question is: how does the environment shape the learner and, in turn, how does the learner influence the learning environment? In other words: what is the transactional relationship of the learning environment? This involves understanding the motivations of the learner with respect to the time and place in which s/he acquires knowledge (Lave and Wenger, 1991)[23]. The learning environment in this context is composed of the learner, other students and teachers and the physical environment. Twenty-first century learning environments are envisioned as places where the learner is engaged in self-directed and co-operative learning activities, and the physical environment is planned so that it can be routinely re-organized to mediate learning (Partnership for 21st Century Skills, 2002)[24]. Therefore, 20th century constructivist concepts which view the learner as active and the learning environment as passive should be replaced with a new perspective. Practice theory recognizes that the learner and the learning environment are active (Dent-Read and Zukow-Goldring, 1997)[25]. In the constructivist setting, students learn from their own discoveries, whereas with practice theory learners are transformed and shaped by their transactions alongside others and their physical settings. 


\section{Practice Theory and Education [26]}

This theory challenges how we think about 'practice', examining what it means across different fields and sites. It is organized into four themes: discursive practices; practice, change and organizations; practicing subjectivity; and professional practice, public policy and education. Contributors to the collection engage and extend practice theory by drawing on the legacies of diverse social and cultural theorists, including Bourdieu, de Certeau, Deleuze and Guattari, Dewey, Latour, Marx, and Vygotsky, [27] by building on the basis of theoretical trajectories of contemporary authors such as Karen Barad, Yrjo Engestrom, Andreas Reckwitz, Theodore Schatzki, Dorothy Smith, and Charles Taylor. The proximity of ideas from different fields and theoretical traditions in the book highlight key matters of concern in contemporary practice thinking, including the historicity of practice; the nature of change in professional practices; the place of discursive material in practice; the efficacy of refiguring conventional understandings of subjectivity and agency; and the capacity for theories of practice to disrupt conventional understandings of asymmetries of power and resources. Their juxtaposition also points to areas of contestation and raises important questions for future research. Practice Theory and Education will appeal to postgraduate students, academics and researchers in professional practice and education, and scholars working with social theory. It will be of particular interest to those who wish to move beyond the limiting configurations of practice found in contemporary neoliberal, new managerialist and narrow representationalist discourses.

\section{Integrating Technology Fully Into The Learning Environment}

In terms of innovation, the 21st century learning ideals are not so different from Reggio Emilia and Montessori pedagogies. Both aspired to engage learners in activities with a variety of tools. Furthermore, these alterative programs are places where faculty and students are motivated to extend their development beyond their current level of knowledge. On the whole, the goals foster critical thinking, social skills (through co-operative activities) and selfdirected work. Whereas Reggio Emilia viewed the physical environment as the "third teacher" who guides learning, Montessori recognized that it must be prepared with tools to promote learning opportunities. Similarly, 21st century learning environments are using today's tools (i.e. information technologies) which are believed to guide the learner and lead development (Vygotsky, 1978) [27].Montessori developed teaching tools that encouraged learners to explore their environments through self-directed and co-operative learning activities. At the time, this was an innovative and modern approach. Since the early 1900s, technology, beginning with film, then radio, television and video were brought into the learning environment (Oliver, 2004)[28]; currently, the computer, tablets and SMART boards have been introduced into instructional settings. However, none of these past or current technologies are being fully integrated into educational programs as was anticipated (Weiss, 2007[29]). One reason for this is that the design of the physical environment does not support the integration of technology (Oliver and Lippman, 2007; Weiss, 2007)[30]. How can the design professional envision a space that includes technology if the advantages and constraints of this tool have not been fully evaluated in relation to the pedagogy of the place? Otherwise put, learning environments should be programmed, planned and designed to support the intended learning activities. In addition, the spatial design of the learning environment, especially in American education, is structured around the classroom. On the whole, these spaces have not changed for decades.

\section{Revising Of Educational Process Modeling:-}

This revising section introduces the conceptual basis of teaching/learning process and illustrates its realistic interactive modeling via two subsections: $(A, B)$ as follows. At the subsection $A$, a generalized brief overview of the block diagram describing interactive teaching/learning process is given. A detailed mathematical formulation introduced at subsection $B$, at either bidirectional communication between a teacher and his learners (supervised) or self-organized (unsupervised). That self-organized could be considered as learning by interaction with environment; either Kohonen or Hebbian paradigms [4][5].

\section{Modeling of Interactive Learning Processes}

Referring to Fig. 1, it illustrates a general view of a teaching model qualified to perform simulation of above mentioned brain functions. Inputs to the neural network teaching model are provided by environmental stimuli (unsupervised learning). However, correction signal(s) in the case of learning with a teacher given by output response(s) of the model that evaluated by either the environmental conditions (unsupervised learning) or by supervision of a teacher. Furthermore, the teacher plays a role in improving the input data (stimulating learning pattern) by reducing the noise and redundancy of model pattern input. That is in accordance with tutor's experience while performing either conventional (classical) learning or Computer Aided Learning. Consequently, he provides the model with clear data by maximizing its signal to noise ratio [22]. Conversely, in the case of unsupervised/self- 
organized learning, which is based upon Hebbian rule [31], it is mathematically formulated by equation (7) presented at next subsection $(B)$.

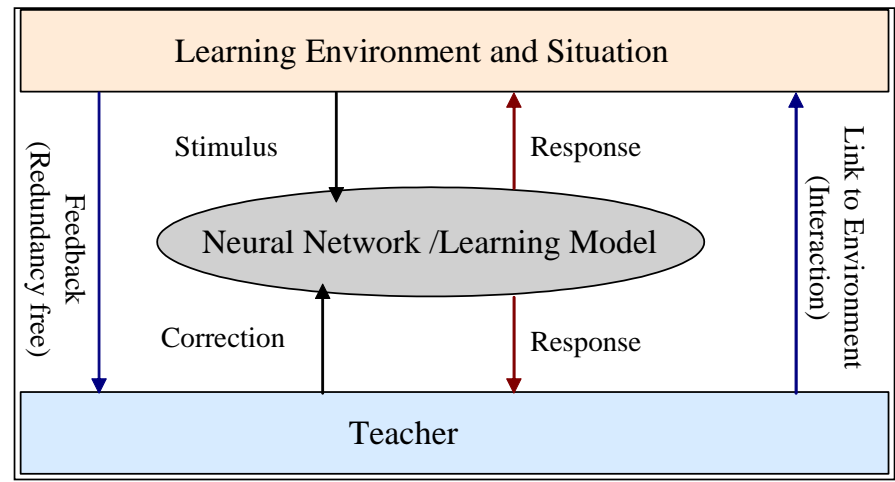

Fig. 1:- Simplified view for interactive learning process \{Adapted from [8]\}.

The presented model given in Fig. 2, simulates realistically the two diverse learning paradigms. It presents both paradigms: by interactive supervised learning/ teaching process, as well as other self-organized (autonomous) learning process. By more details, firstly is concerned with classical (supervised by a tutor) learning observed in our classrooms (face to face tutoring). Accordingly, this paradigm proceeds interactively via bidirectional communication process between a teacher and his learners (supervised learning) [5]. However, the second other learning paradigm performs self-organized (autonomously unsupervised) interactive learning process [32].

Mathematical Formulation of Interactive learning

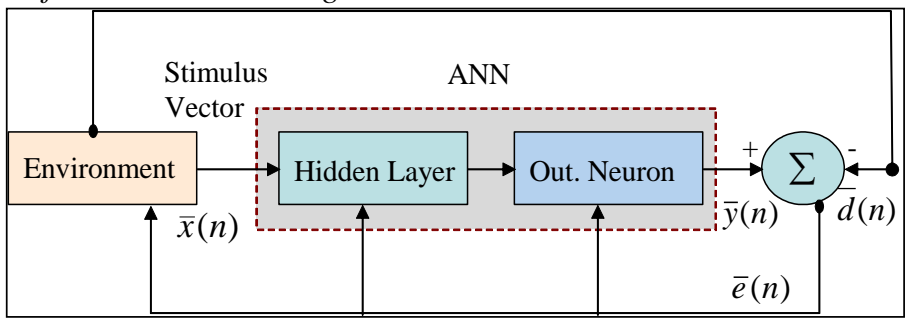

Figure 2:- Generalized ANN block diagram simulating two diverse learning paradigms adapted from [5].

Referring to above Figure 2; the error vector $\bar{e}(n)$ at any time instant (n) observed during learning processes is given by:

$\bar{e}(n)=\bar{y}(n)-\bar{d}(n)$

Where ...... is the error correcting signal that adaptively controls the learning process, $\bar{y}(n) \ldots \ldots$ is the output obtained signal from ANN model, and $\bar{d}(n) \ldots \ldots$ is the desired numeric value(s). Moreover, the following four equations are deduced:

$$
\begin{aligned}
& V_{\mathrm{k}}(n)=X_{j}(n) W_{k j}^{T}(n) \\
& Y_{\mathrm{k}}(n)=\varphi\left(V_{\mathrm{k}}(n)\right)=\left(1-\mathrm{e}^{-\lambda V_{\mathrm{k}}(n)}\right) /\left(1+\mathrm{e}^{-\lambda V_{\mathrm{k}}(n)}\right) \\
& e_{\mathrm{k}}(n)=\left|d_{\mathrm{k}}(n)-y_{\mathrm{k}}(n)\right| \\
& W_{\mathrm{kj}}(n+1)=W_{\mathrm{kj}}(n)+\Delta W_{\mathrm{kj}}(n)
\end{aligned}
$$

Where $X$ is input vector and $W$ is the weight vector. $\varphi$ is the activation function. $Y$ is the output. $e_{\mathrm{k}}$ is the error value and $d_{\mathrm{k}}$ is the desired output. Note that $\Delta W_{\mathrm{kj}}(\mathrm{n})$ is the dynamical change of weight vector value. Above four equations 
are commonly applied for both learning paradigms: supervised (interactive learning with a tutor), and unsupervised (learning though student's self-study). The dynamical changes of weight vector value specifically for supervised phase is given by:

$$
\Delta W_{\mathrm{kj}}(n)=\eta e_{\mathrm{k}}(n) X_{j}(n)
$$

Where $\eta$ is the learning rate value during the learning process for both learning paradigms. At this case of supervised learning, instructor shapes child's behavior by positive/ negative reinforcement Also, Teacher presents the information and then students demonstrate that they understand the material. At the end of this learning paradigm, assessment of students' achievement is obtained primarily through testing results. However, for unsupervised paradigm, dynamical change of weight vector value is given by:

$$
\Delta W_{\mathrm{kj}}(n)=\eta Y_{\mathrm{k}}(n) X_{j}(n)
$$

Noting that $e_{\mathrm{k}}(\mathrm{n})$ equation (6) is substituted by $y_{\mathrm{k}}(\mathrm{n})$ at any arbitrary time instant (n) during the interactive learning process. Referring to Fig.1, the correction signal which provided by a teacher should take into consideration the noisy environmental level inside classrooms(such as noisy crowdedness appears as CPE). In other words, that level is quantitatively measured as signal to noise $(\mathrm{S} / \mathrm{N})$ ratio or equivalently the additive noise power $(\sigma)$ to the ideally sensory clear signal. Consequently, the response time response measured by number of training cycles (n) $\{$ as defined at the subsection in the above $(B)$ by the two equations (6)\&(7) \}. Noting value of (n) should have been increased until reaching learning convergence instant, when

$\Delta W_{\mathrm{kj}}(n)=0$

That above condition given by equation (8), could be fulfilled only if the desired output learning has been obtained after some number of training cycles (response time) in fulfillment of the two equations (6) \& (7). Therefore, the impact of interactive noisy CPE on learning convergence time has been presented at TABLE 1 which derived from given two figures Fig.3 \& Fig.4. All are adapted from the simulation results findings introduced at [22]. Conclusively, it is observed during interactive learning process that: teaching/learning environment with decreasing $\mathrm{S} / \mathrm{N}$ ratio results in decreasing of learning rate parameter value $\eta$. That explicitly computed as noise power value $(\sigma)$

Table.I:- The Effect Of Cocktail Party Noisy Environment On Learning Convergence Time, \{Adapted From [22]\}

\begin{tabular}{|l|l|l|l|}
\hline Signal to Noise Power Ratio of Input Data & 5 & 10 & 20 \\
\hline Noise Power $\sigma$ in Learning Environment & 0.2 & 0.1 & 0.05 \\
\hline $\begin{array}{l}\text { Convergence Learning } \\
\text { Time (cycles) }\end{array}$ & 85 & 62 & 47 \\
\hline
\end{tabular}

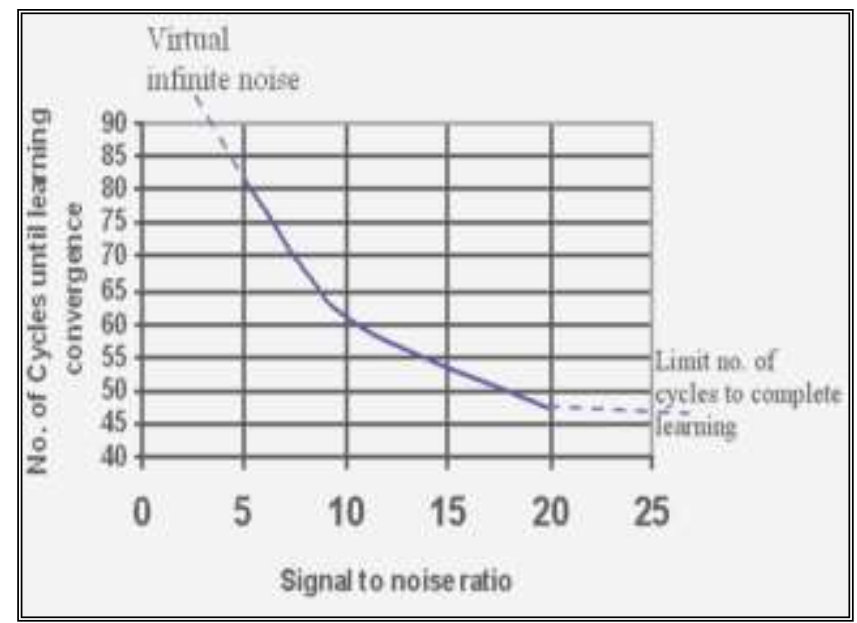

Fig.3:- Graphical presentation of learning performance considering non-properly prepared (noisy) teacher by referring to Table 1. 
Referring to Fig. 4, the three changes of noise power values $\sigma(0.2,0.1$, and 0.05$)$ correspond respectively to noisy contaminated environmental information/data having the values of $\mathrm{S} / \mathrm{N}$ (5, 10, and 20). Interestingly, that by the increasing of $\mathrm{S} / \mathrm{N}$ ratio (more properly prepared teacher) results in well improvement of learning rate parameter value $(\eta)$.

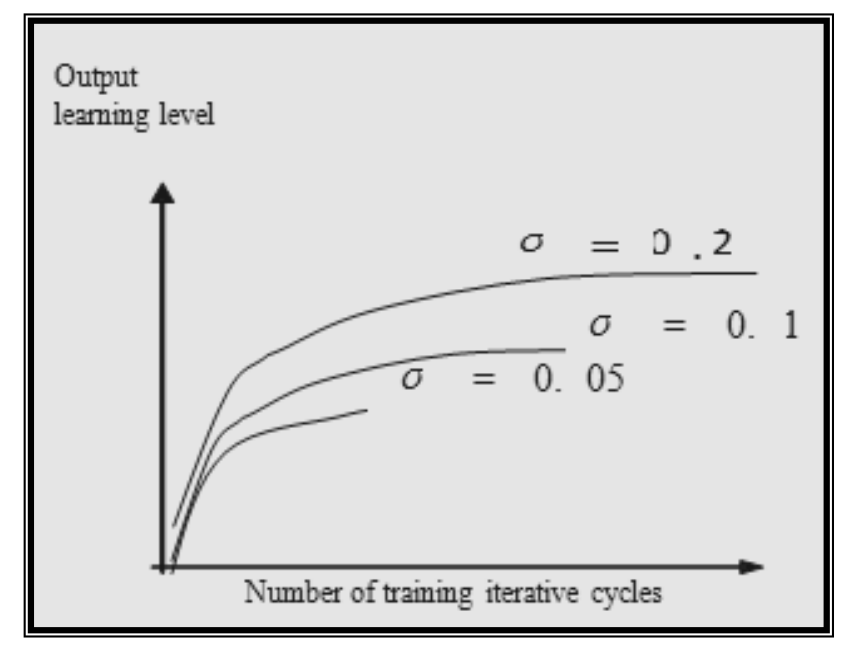

Fig. 4:- relation between noise power $(\sigma)$ that represents non-properly prepared (noisy) learning process convergence, $\{$ Adapted from [22]\}.

\section{Revising Cocktail Party Effect:-}

The Cocktail Party Effect defined as the phenomenon of being able to focus one's auditory attention on a particular stimulus while filtering out a range of other stimuli, as when a partygoer can focus on a single conversation in a noisy room.[33][34] This effect is what allows most people to "tune into" a single voice and "tune out" all others. It may also describe a similar phenomenon that occurs when one may immediately detect words of importance originating from unattended stimuli, for instance hearing one's name in another conversation.[35][36] . Briefly CCP is a challenging problem in auditory perception. Historically, this CCP observed at cocktail party phenomenon, the delineation of which goes back to a classic paper by Cherry in 1953 [37][38]. In this section, it is worthy to address the following issues associated to CCP : (1) human auditory scene analysis, which is a general process carried out by the auditory system of a human listener; (2) insight into auditory perception, which is derived from Marr's vision theory; (3) computational auditory scene analysis, which focuses on specific approaches aimed at solving the machine cocktail party problem; (4) active audition, the proposal for which is motivated by analogy with active vision, and (5) discussion of brain theory and independent component analysis, on the one hand, and correlative neural firing, on the other [39].Interestingly, the ability to maintain a conversation with one person while at a noisy cocktail party has often been used to illustrate a general characteristic of auditory selective attention, namely that perceivers' attention is usually directed to a particular set of sounds and not to others [40][41]. Part of the cocktail party problem involves parsing co-occurring speech sounds and simultaneously integrating these various speech tokens into meaningful units ("auditory scene analysis"). That auditory scene analysis framework to be neurobiologically feasible, it would have to accommodate the ability to switch the focus of attention from one speech signal of interest to another and do so with relative ease [39]. Conclusively, the Cocktail Party Effect phenomenon process is experienced as following one speaker in the presence of another. Such common experience, considered for granted as called: "the cocktail party problem" CPP. It can be trivial experienced process for a normal human (student) listener. From a neurological P.O.V., sounds all enter the ear as one cacophonous roar, but the brain processes all the information and tunes into one sound, such as a person's voice, and filters out the rest [42]. Interestingly, referring to brain functions and anatomical structure, sound and light are processed by different receptors and neural pathways in the brain. However, by considering current knowledge of how auditory and visual stimuli sensations are responding to sound and light respectively. They are represented in the nervous system in similar complexity and that undergo with similar initial processing by the nervous system [43].

\section{Classroom Noise Effect On Students' Academic Achievements:-}

Generally, from the human health point of view, the environmental Noise in elevated sound pressure levels has been indicated as a major risk factor for human health and contaminated noisy learning environmental. However, a great 
part of the noisy crowded population, especially teenagers and young adults have social and personal habits that expose them to learning. Which is analogous to CPE resulting in deteriorated quality of academic achievement due to noisy environmental learning.[44][45][46]. More specifically, in Fig. 5, the noisy effect due to crowdedness (Peoples' Voices) in classrooms represented approximately by [41\%]. In other words, more investigations have shown that excess noise is harmful to the teaching-learning process, since it is distracting, restricts attention level and cognition, and makes listening and understanding the teacher's voice more difficult for the students [47]. By more details, this research work addresses the challenging question: how students could focus on teachers' interactive speaking in noisy overcrowd environment?. From neurological Point of view, all environmental sounds stimulate student's ears and pass to their way via the central brain auditory nervous system. Accordingly, any student is responsible for processing signals of auditory information originated from teachers' speaking signals voice. Which contaminated by various noisy environmental signals' levels, resulting in deterioration of signal to noise ratio values [48]. When discussing the auditory system it is important to understand the difference between the physical mechanism of the ear and the central auditory nervous system in the brain responsible for processing auditory information [49]. The aim of this brief literature review identifies the relationship between noise in schools and educational activities. The environmental noise has direct negative effects on learning, due to distraction and irritability. The sources of these noises vary from those located in the school's external environment to background noise, coming from classrooms as well as the noise generated by the students, during class activities. The authors concluded that noise is part of the causes of learning problems, that different methods are needed to solve this issue in schools and that the appropriate solution is related to the nature of the noise Great part of the studies that aim to measure and assess the consequences of noise in schools focuses mainly on Elementary education. This is possibly due to the fact that the children are learning how to read and write, have less control over their own attention and concentration skills because of their young age, and also because they do not have enough Independence to study by themselves; factors that make the acquisition of written communication more difficult [50]. Recently, two published papers presented some very interesting findings which associated to the impairments of learning environment (learning quality) in classrooms [51][52].

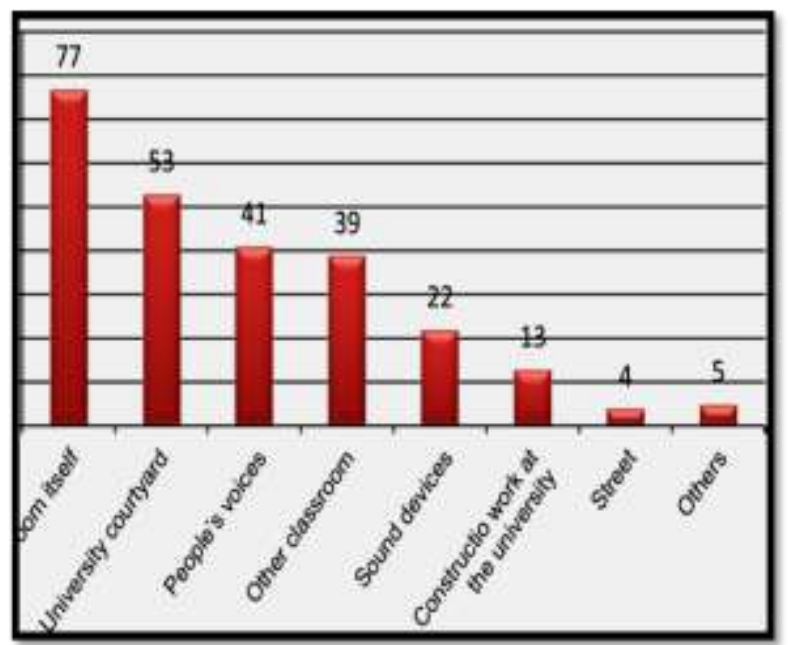

Fig.5:- Illustrates the percentage of noise sources reported by students. Adapted from [44].

\section{Simulation Reults:-}

This simulation results introduced in this section composed of eight figures (Fig.6, Fig.7, Fig.8, Fig.9, Fig.10, Fig.11, Fig.12, and Fig.13). some interesting simulation results presented after taking into account the comparative studies of two essential ANN parameters namely : learning rate and gain factor values. Versus varying neurons' number of the hidden layer associated to self-organized ANN paradigm model. These results revealed the effect of interrelations a among various learning rate values against different values of signal to noisy ratio considering student's selective responsive for focusing attention of considering the contributions of the number of neurons inside any student' brain. Referring to Fig.6, a simplified macro-level flowchart for simulation program is introduced. It describes briefly the algorithmic steps for a suggested realistic simulation program of adopted Artificial Neural Networks' model taking into account the different number of neurons.(\# neurons). 
The three figures (Fig.7, Fig.8, and Fig.9), represented the performance considering different learning rate values, considering Hebbian self-organized learning at different noisy environmental levels and number of contributing neurons. The individual differences of students' selective responsive for focusing attention are represented by various gain factor values. These gain factors illustrated at the two figures (Fig.10, and Fig.13).

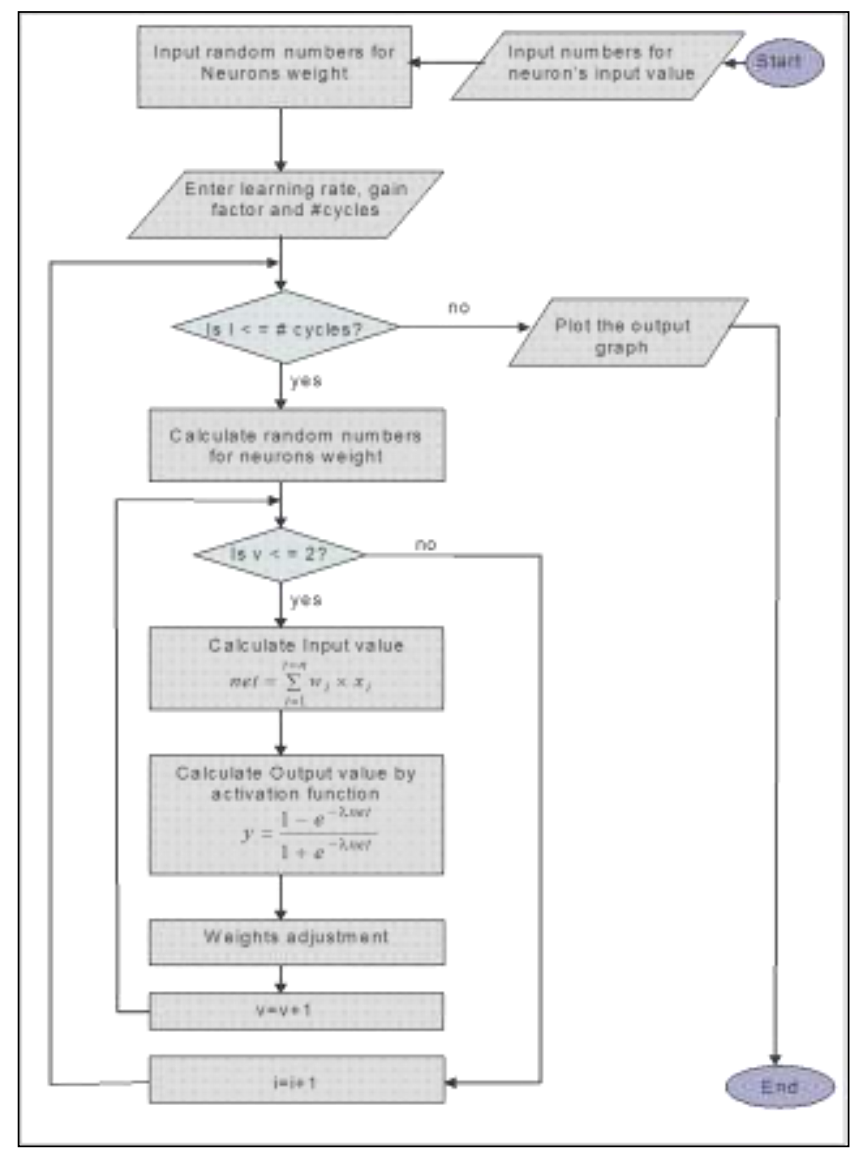

Fig. 6:- A simplified macro level flowchart that describing algorithmic steps for Artificial Neural Networks modeling considering various \# neurons \{Adapted from [51]\}.

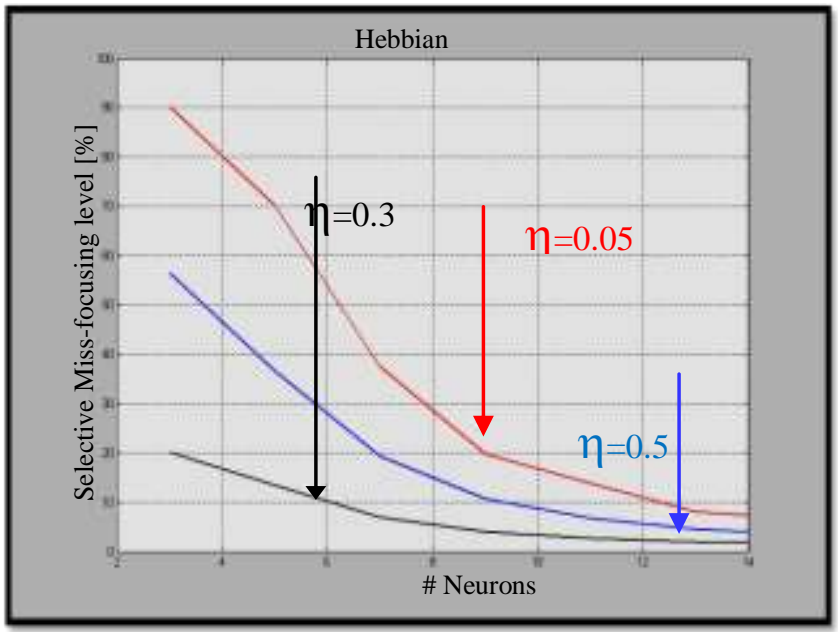

Fig.7:- Illustrates simulated outcomes of Missing Selective Focusing Level presented in percentage [\%] degree of missing lesson focusing versus \# Neurons at different learning rate values $\eta(0.3,0.5$, and 0.05$)$. 


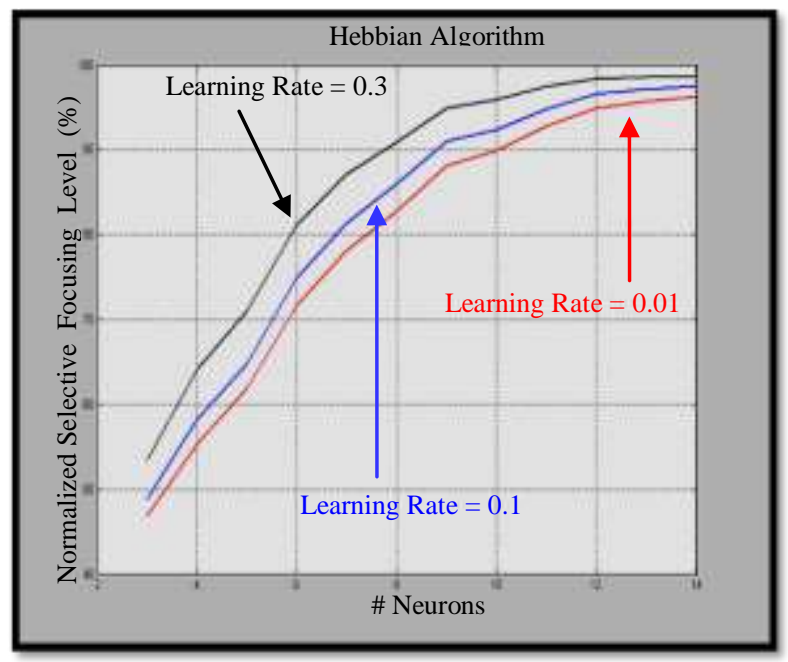

Fig 8:- Illustrates simulated outcomes of Selective Focusing Level presented as percentage degree of lesson focusing versus \# Neurons for different learning rate values $\eta(0.3,0.1$, and 0.01$)$.

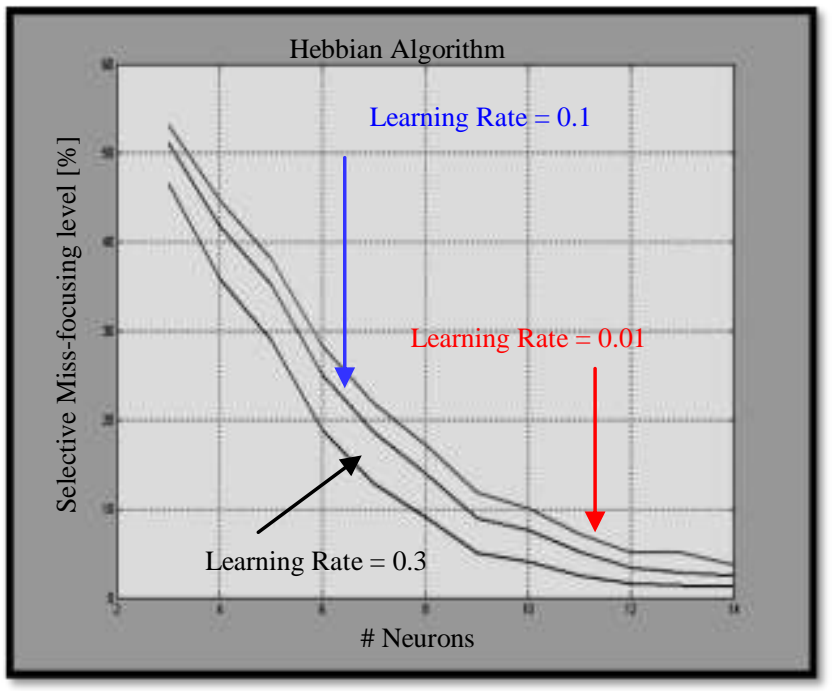

Fig 9:- Illustrate learning performance error-rate with different learning rates when \#cycles= 300 and gain factor $=1$

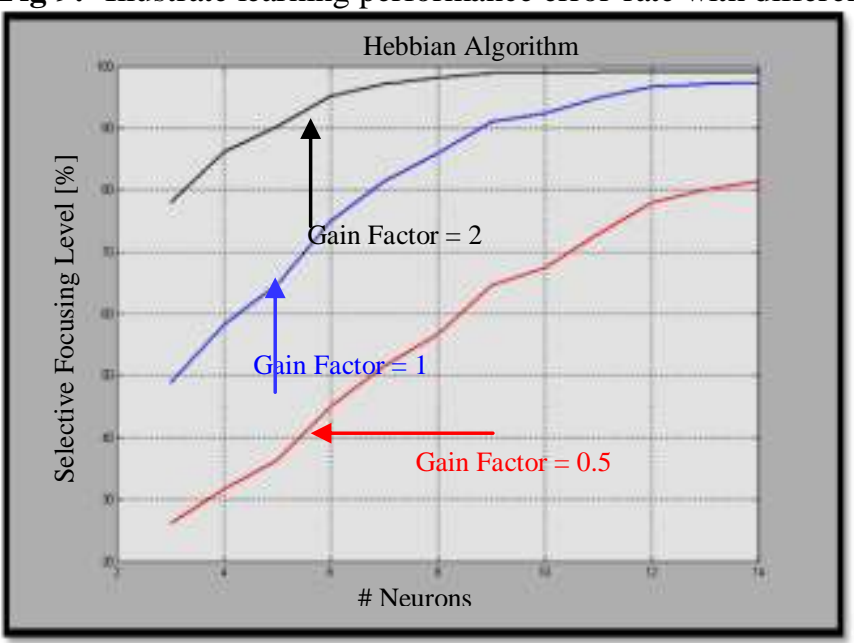

Fig.10:- Learning performance to get accurate solution with different gain factors $0.05,1$, and 2 , while \#cycles $=300$ and Learning Rate $=0.3$ 
Referring to Fig.11, it is worthy to note that statistical variations(on the average) relating learning rate values versus corresponding selectivity convergence (response) time. That time is measured by the number of iteration cycles., obtained output results(of response time) corresponding to the learning rate values $(0.1,0.2,0.4,0.6$, and 0.8$)$, are given respectively, as $(330,170,120,80$, and 40$)$ number of iterative training cycles . Conclusively, convergence time (number of training cycles) is inversely proportional to the corresponding learning rate values as tabulated in TABLE II. Furthermore, it is interestingly noticed that under more noisy environmental conditions, learning rate tends to have lower value. Conversely, creatures performing learning rate improvement by interaction with environment, implies increase of their stored experience. Therefore, such creatures while there interactive learning via environment have become capable of responding spontaneously in optimal manner to input environmental stimuli [8][32]. Furthermore, after running of the suggested realistic simulation program, it results in the set of three distribution curves depicted at Fig. 12. These distributions considered to be in correspondence with the three learning rate values $\square \square(0.05,0.1$, and 0.3$)$ respectively. Noting that the nearness of balance point (at the $\mathrm{x}$-axis) is a suggested measure for degree of exact tuning to understand the speech of one person. Furthermore, after running of the suggested realistic simulation program, it results in the set of three distribution curves at Fig.12., considered three different learning rates and fixed training time (\#cycles $=300) \&$ gain factor $=1$.

TABLE II: THE RELATION BETWEEN LEARNING RATE VALUES AND CONVERGENCE LEARNING TIME

\begin{tabular}{|l|l|l|l|l|l|}
\hline Learning rate Value $(\eta)$ & 0.1 & 0.2 & 0.4 & 0.6 & 0.8 \\
\hline Average Response time & 330 & 170 & 120 & 80 & 40 \\
\hline
\end{tabular}

Number of iteration cycles

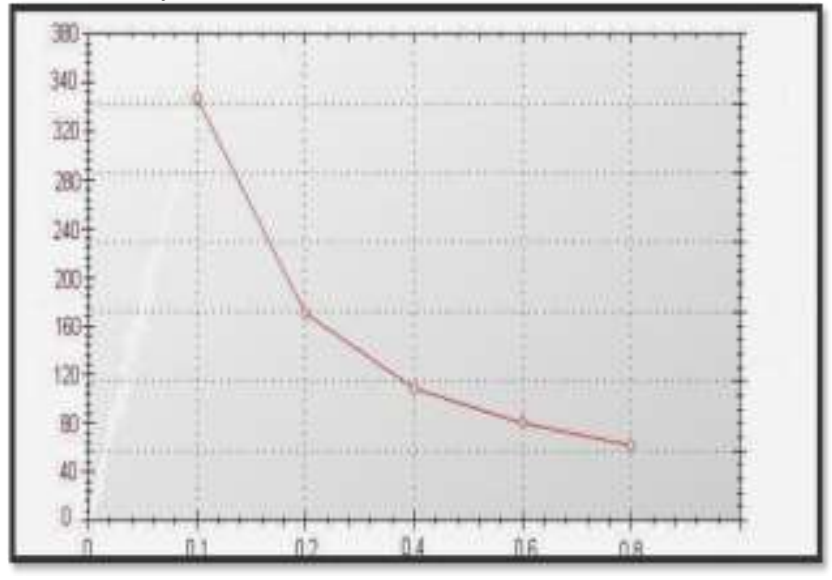

Learning rate values $(\eta)$

Fig. 11:- Illustrates the average of statistical distribution for learning response time (number of iteration cycles) for different learning rate values $\eta$.

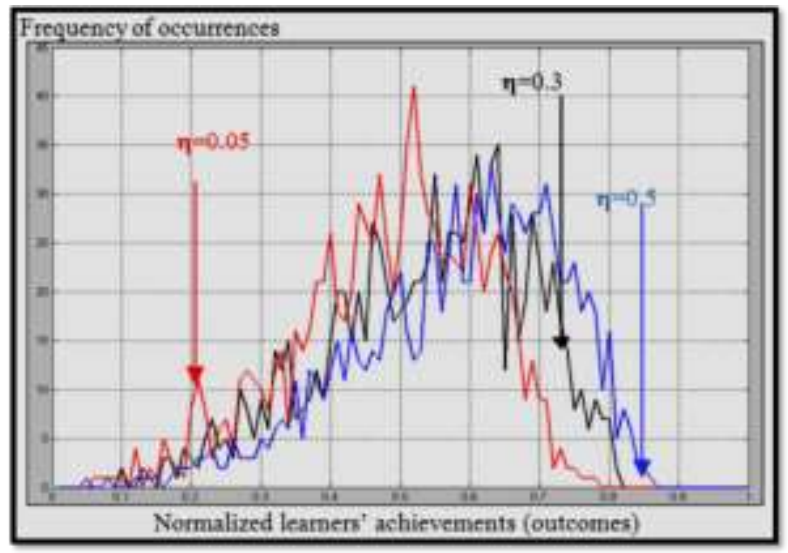

Fig. 12:- The three changes of noise power values $\sigma(0.2,0.1$, and 0.05$)$ innoisy environment considered to be in correspondence with three learning rate values $\eta(0.05,0.3$, and 0.5$)$. 


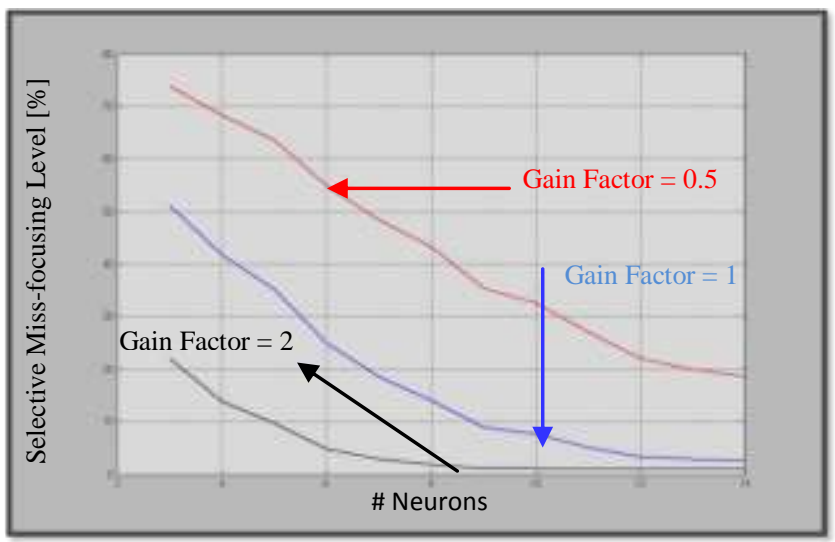

Fig. 13:- Hebbian learning performance for missed focusing versus different gain factor values : $0.5,1$, and 2 , while \#cycles $=300$ and Learning rate $=0.3$.

\section{Conclusions and Disscutions:-}

Considering an arbitrary educational level, any school bears its responsibility to create relevantly enhanced proper learning environment. hat is based on modern skills and knowledge and facilitates students' understanding of the world of technology. That learning environment is regarded as the ensemble of the intellectual, social, and physical environments. Accordingly, schools' responsibility have to take into account students' developed learn performance aside from noisy contaminated (undesirable) impact on created learning environment. This work illustrates clearly the undesirable effect observed of overcrowded population inside classrooms which results in Cocktail Party Problem on deterioration of learning performance phenomenon. Therefore, conversely the ideally noiseless learning environment supporting students' enhanced development into an independent and active learner. Considering the basic values of educational levels and the school's mental attitude, and preserves and refines the traditions of the region and the school community. Accordingly, that learning environment creates principle prerequisites and conditions for acquiring a subject as well as for the development of the student's personality. Interestingly, referring to ANN modeling context, the two parameters: Learning rate and Gain factor are considered by the presented simulated comparative study. Accordingly, interesting simulation results have been obtained by the end conclusion of this work declaring the interrelation between learning rate values versus different noisy levels. As well as, the effect of intrinsic individual children's differences (gain factor values) on selective attention performance is presented. Furthermore, the work illustrates specifically the analogous effect between Artificial Neural Networks modeling of noisy audible data (education in classrooms), versus the noisy physical visual data such as Optical Character Recognition (OCR).The overcrowding in classrooms shown to have negative effect on educational process similar to the noisy learning environmental effect. The interesting results have been obtained indicating an extendable future challenging research. In future, this work is recommended to be extended by more elaborate practical educational field application, in order to investigate systematically both observed educational phenomena presented herein.

\section{REFERENCES:-}

1. White House OSTP Issues Decade of the Brain Report, Maximizing Human Potential: 1990-2000. Available on line at: http://www.neurology.org/content/40/2/321.extract

2. Signal to noise ratio definition, posted by Margaret Rouse. Available on line at: http://searchnetworking.techtarget.com/definition/signal-to-noise-ratio

3. Mariale Hardiman posted on line at: http://www.mona.uwi.edu/cop/sites/default/files/resource/files/The\%20Brain\%20Targeted\%20Teaching\%20Model.pdf

4. Kohonen T. "self-organization and Associative Memory": New York, Springer, 1984.

5. Haykin S., Neural Networks, Englewood Cliffs, NJ: Prentice-Hall, 1999.

6. Hassan M. H. Mustafa "On performance evaluation of brain based learning processes using neural networks," published at 2012 IEEE Symposium on Computers and Communications (ISCC), pp. 000672-000679, 2012 IEEE Symposium on Computers and Communications (ISCC), 2012.

7. Available online at : http://www.computer.org/csdl/proceedings/iscc/2012/2712/00/IS264-abs.html

8. Jayanta Kumar Basu, Debnath Bhattacharyya, Tai-hoon Kim "Use of Artificial Neural Network in Pattern Recognition" International Journal of Software Engineering and Its Applications Vol. 4, No.2, April 2010. 
9. Hassan M. H. Mustafa, S.Mahmoud, Ibrahim H. Assaf, Ayoub Al-Hamadi , Zedan M. Abdulhamid "Comparative Analogy of Overcrowded Effects in Classrooms versus Solving 'Cocktail Party Problem' (Neural Networks Approach)" Published at the International Journal of Engineering Science and Innovative Technology (IJESIT) ISSN: 2319-5967 ISO 9001:2008, Volume 3, Issue 2, March 2014.

10. H. Mustafa, S. Mahmoud, A. Al-Hamadi, Z.M. Abdulhamid, A.M. Al-Bassiouni "ON QUANTIFIED EVALUATION OF NOISY DATA IMPACT ON CHILDREN'S MENTAL DEVELOPMENT USING ARTIFICIAL NEURAL NETWORKS" Published at ICERI2013, the 6th International Conference of Education, Research and Innovation held in Seville (Spain), on the 18th, 19th and 20th of November, 2013. published at ICERI2013 Proceedings. Available on line at: http://library.iated.org/view/MUSTAFA2013ONQ.

11. Ames, C. (1992). Classrooms: Goals, structures, and student motivation. Journal of Educational Psychology, 84(3), 261-271. Available online at: http://dx.doi.org/10.1037/0022-0663.84.3.261.

12. Douglas, R. J., \& Martin, K. A. C. (1991). Opening the gray box. Trends in Neurosciences, 14, $286-293$.

13. Swaminathan,N 2007 " Cognitive Ability Mostly Developed Before Adolescence, NIH Study Says. NIH announces preliminary findings from an effort to create a database that charts healthy brain growth and behavior " Scientific American letter, May 18, 2007.

14. Swaminathan,N "How The Brain Maps Symbols To Numbers" Scientific American letter, October 31, 2007.

15. Borzenko "Neuron mechanism of human languages" Published in IJCNN'09 Proceedings of the 2009 international joint conference on Neural Networks IEEE, NJ, USA C2009 ISBN: 978-1-4244-3549-4.

16. H.M. Hassan ,A. Al-Hamadi, B.Michaelis " Evaluation of Memorization Brain Function Using a Spatiotemporal Artificial Neural Network (ANN) Model" Published at CCCT 2007 conference ,July12-15 ,2007 Orlando, Florida, USA.

17. Mustafa, et. al. "On Assessment of Brain Function Adaptability in Open Learning Systems Using Neural Networks Modeling (Cognitive Styles Approach)", IEEE International Conference on Communications and Information Technology ICCIT-2011, Mar 29, 2011 - Mar 31, 2011, Aqaba, Jordan. Published also at Journal of American Science, 2011: 7(4), http://www.americanscience.org.

18. H.M. Mustafa "Building up bridges for natural inspired computational models across behavioral brain functional phenomena; and open learning systems" A tutorial presented at the International Conference on Digital Information and Communication Technology and its Applications (DICTAP2011), June 2011, Dijon, France.

19. Kandel, E: R. "Small Systems of Neuron“, Scientific American, Sept. 1979, vol.224, pp. 67-79. [12] Marr, D., "A theory of Cereballar cortex", T. Physiol. (London) 202, pp. 437-470,1969.

20. Grossberg, S. (ED.)" Neural Networks and Natural Intelligence” The MIT press, 1988, pp 1-5.

21. H.M.Hassan, Ayoub Al-Hamadi, and F. Al-Mohaya "On Quantifying Learning Creativity Using Artificial Neural Networks (A Nero- physiological Cognitive Approach)" Published at National Conference on Applied Cognitive Psychology held in India , Calcutta , 29 -30 November, 2007.

22. H.M. Hassan" On Simulation of Adaptive Learner Control Considering Students' Cognitive Styles Using Artificial Neural Networks (ANNs)" Published at CIMCA, Austria. 28-30 Nov.2005.

23. Ghonaimy M.A., Al - Bassiouni, A.M. and Hassan, H.M "Leaning of Neural Networks Using Noisy Data". Second International Conference on Artificial Intelligence Applications, Cairo, Egypt, Jan 22-24, 1994.

24. Lave, J. and E. Wenger (1991), Situated Learning, Cambridge University Press, New York.

25. Elkington, J. (1997), Cannibals with Forks: The Triple Bottom Line of 21st Century Business, Capstone Publishing, Oxford.

26. Partnership for 21st Century Skills (2002), "Learning for the 21st century: A report and mile guide for 21 st century skills". Available online at:

27. www.21stcenturyskills.org/images/stories/otherdocs/p21up_Report.pdf.

28. Julianne Lynch, Julie Rowlands, Gale, and Andrew Skourdoumbison "Practice Theory and EducationDiffractive readings in professional practice" .Pub. on: 2016-12-02- by - Routledge-2017-288 pages

29. Vygotsky, L.S. (1978), Thought and Language, MIT Press, Cambridge, MA.

30. Oliver, C. (2004), "Teaching at a Distance: The Online Faculty Work Environment", unpublished dissertation, The City University of New York, New York.

31. Oliver, C. and P.C. Lippman (2007), "Examining space and place in learning environments", paper presented at the CONNECTED International Conference on Design Education, 9-12 July, University of New South Wales, Sydney, Australia.

32. Weiss, A. (2007), "Creating the Ubiquitous Classroom: Integrating Physical and Virtual Learning Spaces, in The International Journal of Learning, Vol. 14, No. 3, www.Learning-Journal.com.

33. D.O. Hebb, "The organization of behaviour", Wiley, New York (1949). 
34. Fukaya, M., et al. Two level Neural Networks: Learning by Interaction with Environment, 1st ICNN, San Diego, 1988.

35. Bronkhorst, Adelbert W. (2000). "The Cocktail Party Phenomenon: A Review on Speech Intelligibility in Multiple-Talker Conditions" (pdf). Acta Acustica united with Acustica. 86: 117-128. Retrieved 2010-04-18.

36. Shinn-Cunningham, Barbara G. (2008). "Object-based auditory and visual attention" (pdf). Trends in Cognitive Sciences. 12: 182-186. doi:10.1016/j.tics.2008.02.003.

37. Wood N, Cowan N (January 1995). "The cocktail party phenomenon revisited: how frequent are attention shifts to one's name in an irrelevant auditory channel?". J Exp Psychol Learn Mem Cogn. 21 (1): 255-60. PMID 7876773. doi:10.1037/0278-7393.21.1.255

38. Conway AR, Cowan N, Bunting MF (June 2001). "The cocktail party phenomenon revisited: the importance of working memory capacity". Psychon Bull Rev. 8 (2): 331-5. PMID 11495122. doi:10.3758/BF03196169.

39. Cherry, E. C. (1953). Some experiments on the recognition of speech, with one and two ears. Journal of the Acoustical Society of America, 25, 975-979.

40. Cherry, E. C., \& Taylor. K. (1954). Some further experiments upon the recognition of speech, with one and, with two ears. Journal of the Acoustical Society of America, 26, 554-559.

41. S. Haykin, and Zhe Chen "The Cocktail Party Problem" Adaptive Systems Lab, McMaster University, Hamilton, Ontario, Canada L8S 4K1.

42. Source: Cell "Solving the 'Cocktail Party Problem': How We Can Focus On One Speaker in Noisy Crowds", Date: March 6, 2013. Available online at: http://www.sciencedaily.com/releases/2013/03/130306134218.htm.

43. J.Hamilton Tuning in to the Brain's 'Cocktail Party Effect', Date: November 23, 2010 2:33 PM Available online at:http://www.npr.org/2010/11/23/131545319/go-wild-birds-bats-share-in-cocktail-party-effect.

44. [2] K. Carollo "The Cocktail Party Effect: How We Tune In to One Person at a Loud Party" Published on Apr 18, 2012. Available online at:http://abcnews.go.com/blogs/health/2012/04/18/the-cocktail-party-effect-how-wetune-in-to-one-person-at-a-loud-pa rty/.

45. [3] K. M Visscher, Elina Kaplan, Michael J Kahana, and Robert Sekuler "Auditory Short-Term Memory Behaves Like Visual Short-Term Memory" Published: February 20, 2007 Available online at: http://www.plosbiology.org/article/info\%3Adoi\%2F10.1371\%2Fjournal.pbio.0050056.

46. Servilha EAM, and Delatti MA " College students' perception of classroom noise and its consequences on learning quality" Published at Audiol Commun Res. 2014;19(2):138-44. Available online at: http://dx.doi.org/10.1590/S2317-64312014000200007.

47. Lacerda ABM, Gonçalves CGO, Zocoli AMF, Diaz C, Paula K. Os hábitos auditivos e comportamento de adolescentes diante das atividades de lazer ruidosas. Rev CEFAC. 2011;13(2):322-9. Available online at: http://dx.doi. org/10.1590/S1516-18462010005000129

48. Knobel KAB, Lima MCMP. Knowledge, habits, preferences, and protective behavior in relation to loud sound exposures among Brazilian children. Int J Audiol. 2012;51 Supl 1:12-9. Available online at:

49. http://dx.doi.org/10.3109/14 992027.2011.637307

50. Ramma L. Knowledge and attitudes of teachers regarding the impact of classroom acoustics on speech perception and learning. S Afr J Commun Disord. 2009;56:35-47.

51. Helena Karabulut "The Neuro-Building Blocks of Learning: Improving School Readiness and Overcoming Learning Difficulties" Journal of Education and Future, year: 2013, issue: 4, 1 - 15

52. Hannah, Ryan, "The Effect of Classroom Environment on Student Learning" (2013). Honors Theses. Paper 2375. Western Michigan University Scholar Works at WMU Available online at: http://scholarworks.wmich.edu/honors_theses/?utm_source=scholarworks.wmich.edu\%2Fhonors_theses\%2F23 75\&utm_medium=PDF\&utm_campaign=PDFCoverPages.

53. Klatte $\mathrm{M}$, Lachmann $\mathrm{T}$, Meis $\mathrm{M}$. Effects of noise and reverberation on speech perception and listening comprehension of children and adults in a classroom-like setting. Noise Health. 2010;12(49):270-82.Available online at: http://dx.doi. org/10.4103/1463-1741.70506.

54. Hassan. M. Mustafa and Ayoub Al-Hamadi "An Overview on Classrooms' Academic Performance Considering: Non-properly Prepared Instructors, Noisy Learning Environment, and Overcrowded Classes (Neural Networks' Approach)" . Published in International Journal of Learning and Teaching (IJLT) Vol.2, No.2, 2016.

55. Hassan. M. Mustafa and Ayoub Al-Hamadi "On Comparative Analogy of Academic Performance Quality Regarding Noisy Learning Environment versus Non-properly Prepared Teachers Using Neural Networks' Modeling" .Published in International Journal of Information and Education Technology, Vol. 6, No. 12, December 2016. 